\title{
ARTICLE
}

\section{One order of magnitude faster phase change at reduced power in Ti-Sb-Te}

Min Zhu ${ }^{1, \star}$, Mengjiao Xia ${ }^{1, \star}$, Feng Rao ${ }^{1}$, Xianbin $\mathrm{Li}^{2}$, Liangcai $\mathrm{Wu}^{1}$, Xinglong $\mathrm{Ji}^{1}$, Shilong $\mathrm{Lv}^{1}$, Zhitang Song ${ }^{1}$, Songlin Feng ${ }^{1}$, Hongbo Sun $^{2} \&$ Shengbai Zhang ${ }^{2,3}$

To date, slow Set operation speed and high Reset operation power remain to be important limitations for substituting dynamic random access memory by phase change memory. Here, we demonstrate phase change memory cell based on $\mathrm{Ti}_{0.4} \mathrm{Sb}_{2} \mathrm{Te}_{3}$ alloy, showing one order of magnitude faster Set operation speed and as low as one-fifth Reset operation power, compared with $\mathrm{Ge}_{2} \mathrm{Sb}_{2} \mathrm{Te}_{5}$-based phase change memory cell at the same size. The enhancements may be rooted in the common presence of titanium-centred octahedral motifs in both amorphous and crystalline $\mathrm{Ti}_{0.4} \mathrm{Sb}_{2} \mathrm{Te}_{3}$ phases. The essentially unchanged local structures around the titanium atoms may be responsible for the significantly improved performance, as these structures could act as nucleation centres to facilitate a swift, low-energy order-disorder transition for the rest of the Sb-centred octahedrons. Our study may provide an alternative to the development of high-speed, low-power dynamic random access memory-like phase change memory technology.

\footnotetext{
${ }^{1}$ State Key Laboratory of Functional Materials for Informatics, Shanghai Institute of Micro-system and Information Technology, Chinese Academy of Sciences, Shanghai 200050, China. ${ }^{2}$ State Key Laboratory on Integrated Optoelectronics, College of Electronic Science and Engineering, Jilin University, Changchun 130012, China. ${ }^{3}$ Department of Physics, Applied Physics, \& Astronomy, Rensselaer Polytechnic Institute, Troy, New York 12180, USA. * These authors contributed equally to this work. Correspondence and requests for materials should be addressed to F.R. (email: fengrao@mail.sim.ac.cn) or to X.L. (email: lixianbin@jlu.edu.cn) or to Z.S. (email: ztsong@mail.sim.ac.cn).
} 
$\mathrm{P}$ hase change memory (PCM) is one of the promising candidates for 'universal memory', based on the rapidly reversible phase transition between amorphous (a-) and crystalline (c-) states of chalcogenide alloys and accompanied large electrical contrast ${ }^{1,2}$. To melt a PCM material and quench it into the a-state (Reset operation), an electrical pulse of large magnitude and short duration is required. In contrast, to realize the c-state (Set operation), a smaller magnitude and longer duration pulse is needed to heat the material to a temperature between crystallization temperature $\left(T_{\mathrm{c}}\right)$ and melting point $\left(T_{\mathrm{m}}\right)$. Because of its low cost, high reliability and compatibility with the complementary metal-oxide semiconductor (CMOS) technology, $\mathrm{Ge}_{2} \mathrm{Sb}_{2} \mathrm{Te}_{5}$ (GST)-based PCM has already been applied to mobile electronics, replacing NOR flash memory (Micron announces availability of PCM for mobile devices- http://investors.micron. $\mathrm{com} /$ releasedetail.cfm?ReleaseID $=692563,2012)$. However, the slow Set speed and high Reset power remain to be important limitations for substituting DRAM by PCM, as the DRAM requires rigorously $<10 \mathrm{~ns}$ access time and $10^{-11} \sim 10^{-12} \mathrm{~J}$ program energy ${ }^{3}$.

Many efforts have been made to increase the operation speed and/or decrease the power requirement to operate the PCM. These include the following: (I) confined small cell: confined dashtype cell $(7.5 \mathrm{~nm}$ in width) of Sb-rich GST can reach $30 \mathrm{~ns}$ Set speed with $>10^{11}$ cycles $^{3,4}$. (II) Initialization electrical pulse: a weak incubation voltage pulse $(\sim 0.3 \mathrm{~V}$ at $10 \mathrm{~ns})$ on GST can help to pre-form fourfold membered atomic rings, which are the precursors for distorted octahedrons in rocksalt c-GST ${ }^{5,6}$. (III) Diminishing grain size: as the grain size of the GST is reduced from 17 to $13 \mathrm{~nm}$, the Set time can be reduced from 44 to $40 \mathrm{~ns}^{7,8}$. In contrast, reducing the Reset power can not only prolong PCM cell life to compete with DRAM $\left(>10^{15} \text { cycles }\right)^{3}$, but can also effectively lower the standards for drive capability of gating device to achieve high-density integration ${ }^{9,10}$. However, because of the high $T_{\mathrm{m}}\left(600-630^{\circ} \mathrm{C}\right)^{11}$ for the GeTe-Sb $\mathrm{Te}_{3}$ pseudobinary alloys, merely regulating the composition seems not to be very effective. All the methods above inadvertently increase the design and fabrication difficulties of the DRAM-like PCM. This prompts us to explore alternative and simpler approaches to increase the working speed while reducing the working power.

In the present work, the concept of introducing robust pinning sites into $\mathrm{Sb}_{2} \mathrm{Te}_{3}$ (ST) via Ti doping, which forms metal-centred or Ti-centred atomic motifs (TCAMs), is proposed. The TCAM describes a local atomic configuration where the $\mathrm{Ti}$ atom occupies the central site of a distorted or defective octahedron and the $\mathrm{Te}(\mathrm{Sb})$ atoms occupy the vertex sites. With this approach, at least one order of magnitude faster Set speed and as low as one-fifth of the Reset energy were achieved on Ti-doped ST cell compared with those of GST cell with the same size.

The rationale to suggest TCAM is as follows: in GST, the widely accepted phase change models concern with nucleation mechanisms that are facilitated through either a cavity-supported reorientation of four-membered ring structures ${ }^{12-14}$ or a reconfiguration of tetrahedrally bonded Ge atoms to octahedrally bonded $\mathrm{Ge}$ atoms ${ }^{15,16}$. Both models involve considerable atomic rearrangements. To make the process easier, it may be beneficial to pin the local bonding environment of cation $M$ in an octahedral configuration in M-Sb-Te alloy. This way, local rearrangements of the atoms, as well as the Sb-centred motifs adjacent to the M-centred motifs, can be made in favour of the octahedral alignment for long-range order. It means TCAMs should commonly exist in both a- and c- phases. Less configuration changes make it possible for the M-centred local structures act as intrinsic nucleation centres to lower the energy barriers required for phase transition. The TCAM represents a phase transition mechanism qualitatively different from that of GST.

\section{Results}

Comparison of Set operation speed. The cross-section structure of a T-shaped PCM cell with a diameter $(D)$ of $190 \mathrm{~nm}$ bottom electrode contact (BEC) is presented in Fig. 1a, which is fabricated by the $0.13 \mu \mathrm{m}$ CMOS technology. As the magnitude of applied voltage pulse reaches $1.3 \mathrm{~V}$, the $\mathrm{Ti}_{0.4} \mathrm{Sb}_{2} \mathrm{Te}_{3}$ (TST) cell shows a Set speed of $\sim 6 \mathrm{~ns}$, while the GST cell with the same size requires $\sim 75 \mathrm{~ns}$ even at $1.6 \mathrm{~V}$ (see Fig. $1 \mathrm{~b}$ and Supplementary Fig. 1). Namely one order of magnitude faster Set speed can be achieved. The TST cell is reasonably stable, too, as after $\sim 10^{7}$ cycles (see Fig. 1a and Supplementary Fig. 2), Ti, Sb and Te are still uniformly distributed without any sign of severe segregation.

Comparison of Reset operation power. Figure 2 shows that even the Reset energy of the TST cell with $D=190 \mathrm{~nm} \mathrm{BEC}$ $\left(3.12 \times 10^{-9} \mathrm{~J}\right)$ can be noticeably less than that of the GST cell with much smaller $D=80 \mathrm{~nm}$ BEC $\left(4.20 \times 10^{-9} \mathrm{~J}\right)$, and a substantial reduction $(\sim 78 \%)$ of Reset energy is achieved on TST cell with the same $D=80 \mathrm{~nm}$ BEC $\left(0.95 \times 10^{-9} \mathrm{~J}\right)$ as GST cell. Inset in Fig. 2 also shows an $82 \%$ reduction of Reset current obtained on the TST cell, compared with the GST cell with the same $D=80 \mathrm{~nm}$ BEC.

Such swift and low-energy switching features, at the same time, do not affect the stability of both the Set and Reset data states of the TST cell. In fact, the data retention (Supplementary Fig. 3)

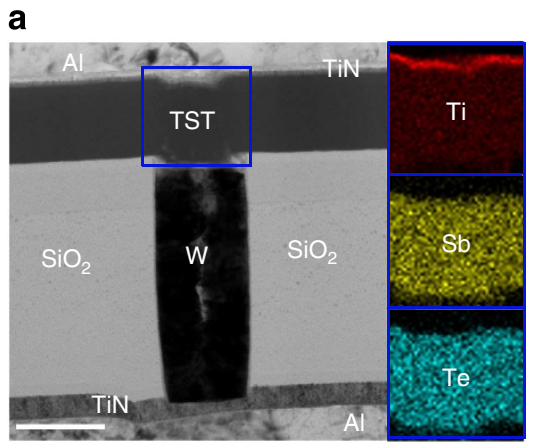

b

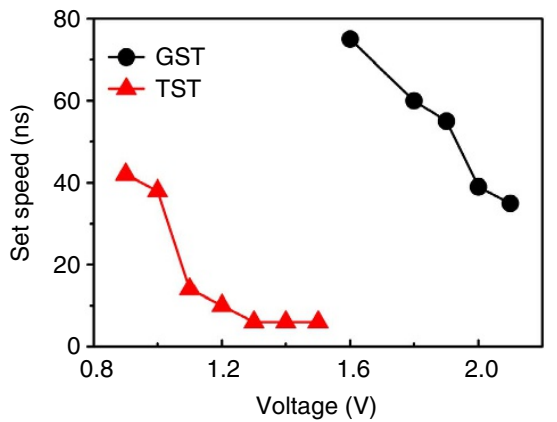

Figure 1 | T-shaped phase-change memory cell and Set operation speed comparison. (a) Cross-sectional TEM image of a T-shaped phase-change memory cell. The scale bar corresponds to $200 \mathrm{~nm}$. The diameter (D) of the W bottom electrode contact (BEC) is $190 \mathrm{~nm}$. The thicknesses for the Al top electrode, TiN layer and $\mathrm{Ti}_{0.4} \mathrm{Sb}_{2} \mathrm{Te}_{3}$ (TST) film are $\sim 300, \sim 15$ and $\sim 170 \mathrm{~nm}$, respectively. Insets show element mapping for Ti, Sb and Te after $\sim 10^{7}$ Set-Reset cycles. (b) Set operation speeds for the TST- and $\mathrm{Ge}_{2} \mathrm{Sb}_{2} \mathrm{Te}_{5}$ (GST)-based PCM cells with the same D=190 nm W BEC. 
and resistance drift (Supplementary Fig. 4) properties of the TST cell are comparable to those of the GST cell. We thus expect that once the fabrication and programming methods are optimized, the overall device performance will be improved further, ensuring future DRAM-like device applications.

Homogeneous phase and lower melting point of TST. In-situ $\mathrm{X}$-ray diffraction (XRD) is used to characterize 200-nm thick ST

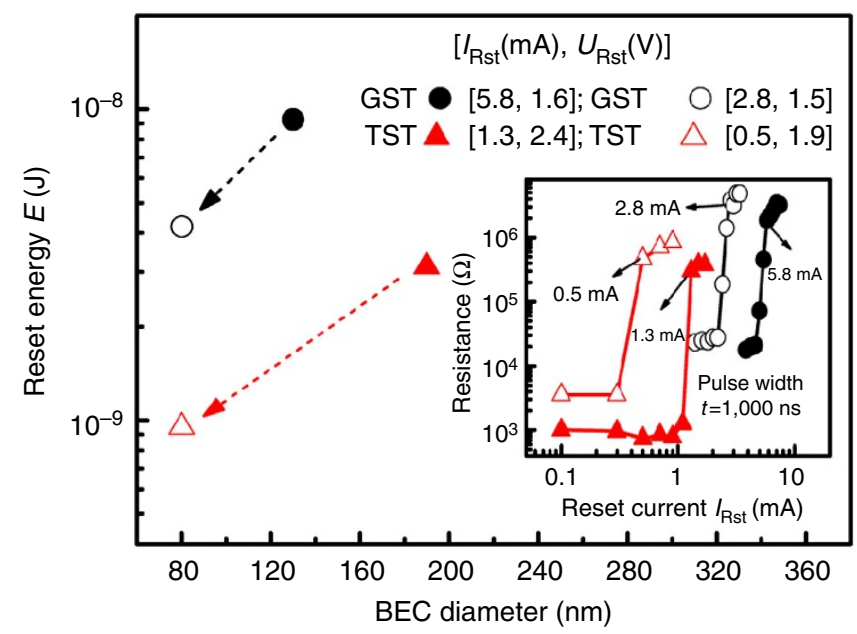

Figure 2 | Reset performance comparison for $\mathrm{Ge}_{2} \mathrm{Sb}_{2} \mathrm{Te}_{5}$ and $\mathrm{Ti}_{0.4} \mathrm{Sb}_{2} \mathrm{Te}_{3}$ phase-change memory cells. Reset energy $(E)$ as a function of bottom electrode contact (BEC) diameter (D) for the $\mathrm{Ge}_{2} \mathrm{Sb}_{2} \mathrm{Te}_{5}$ (GST) and $\mathrm{Ti}_{\mathrm{O} .4} \mathrm{Sb}_{2} \mathrm{Te}_{3}$ (TST) cells. Inset shows resistance versus Reset current $\left(I_{\mathrm{Rst}}\right)$ curves with a fixed pulse width of 1,000 ns $(t)$. Transient Reset voltage $\left(U_{\text {Rst }}\right)$ across the cell is recorded once the Reset state is reached. The input Reset energy can thus be calculated as $E=I_{\text {Rst }} \cdot U_{\text {Rst }} \cdot t$. The Reset energies are $(0.95,3.12,4.20,9.28) \times 10^{-9} \mathrm{~J}$ for TST $(D=80 \mathrm{~nm})$, TST

$(D=190 \mathrm{~nm}), \mathrm{GST}(D=80 \mathrm{~nm})$ and GST $(D=130 \mathrm{~nm})$ cells, respectively.

a
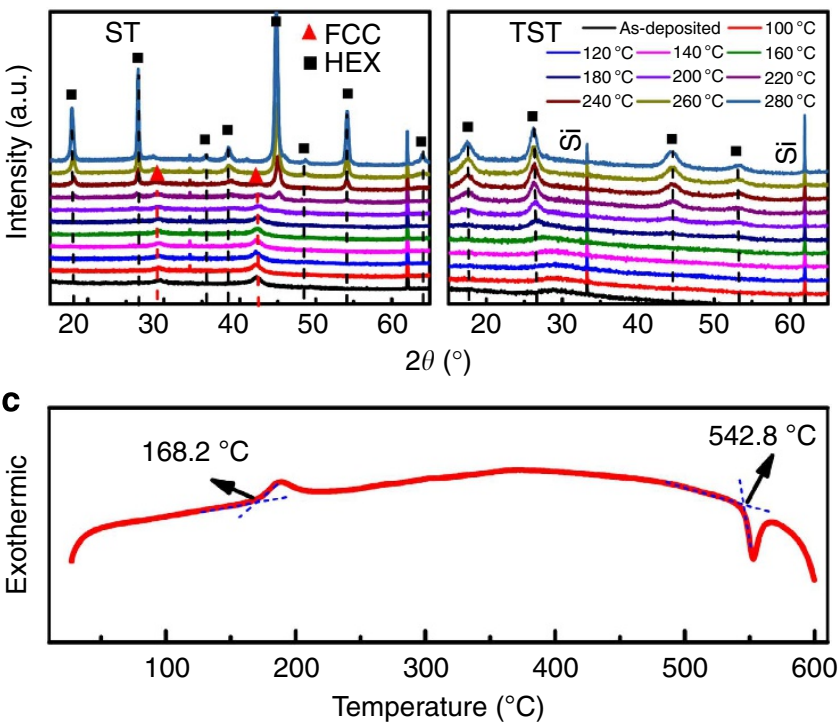

Figure 3 | The in situ X-ray diffraction and differential scanning calorimetry results of $\mathbf{T i}_{\mathbf{0 . 4}} \mathbf{S b}_{\mathbf{2}} \mathbf{T e}_{\mathbf{3}}$. In situ X-ray diffraction curves of (a) $\mathrm{Sb}_{2} \mathrm{Te}_{3}$ (ST) and (b) $\mathrm{Ti}_{0.4} \mathrm{Sb}_{2} \mathrm{Te}_{3}$ (TST) films at different temperatures. (c) Differential scanning calorimetry curve of TST material. The heating rate is $10^{\circ} \mathrm{C} \mathrm{min}^{-1}$. The curve shows an exothermic peak of crystallization as well as an endothermic peak of melting. and TST films on Si substrate, as shown in Fig. 3a,b. For ST, the as-deposited film is partially crystallized because of its low $T_{\mathrm{c}}$. The FCC (face-centred cubic) to HEX (hexagonal) transition takes place at $220^{\circ} \mathrm{C}$ (ref. 17). For TST, the as-deposited film is amorphous. At above $160^{\circ} \mathrm{C}$, it starts to crystallize. While the diffraction peaks are similar to HEX-ST, they are broader, implying an obvious inhibition of grain growth due to Ti doping. No peaks belonging to the $\mathrm{Te}$, $\mathrm{Ti}-\mathrm{Sb}$, or $\mathrm{Ti}-\mathrm{Te}$ phases were observed, which also indicates that the c-TST is a homogeneous phase. Differential scanning calorimetry (DSC) study (Fig. 3c) reveals the melting process of TST commencing at $542.8^{\circ} \mathrm{C}$ $\left(=T_{\mathrm{m}}\right)$. Note that $T_{\mathrm{m}}$ for TST is about $75^{\circ} \mathrm{C}$ lower than that for GST $\left(616^{\circ} \mathrm{C}\right)^{11}$. Therefore, less Reset energy is required for the TST cell of similar dimensions.

\section{Discussion}

Usually, uniformity is an important factor for PCM cyclability; indeed, the lattice parameters $(a)$ of GeTe and $\mathrm{Sb}_{2} \mathrm{Te}_{3}$ constructing the flagship $(\mathrm{GeTe})_{\mathrm{n}}\left(\mathrm{Sb}_{2} \mathrm{Te}_{3}\right)_{\mathrm{m}}$ alloys only deviate slightly $(\sim 2.2 \% \text { for HEX lattice and } \sim 1.0 \% \text { for rocksalt lattice })^{18}$. In contrast, $\mathrm{Si}-\mathrm{Te}, \mathrm{Al}-\mathrm{Te}$ and $\mathrm{Cu}-\mathrm{Te}$ alloys all have noticeably larger lattice mismatch with $\mathrm{Sb}_{2} \mathrm{Te}_{3}$. Not surprisingly, their incorporation into $\mathrm{Sb}_{2} \mathrm{Te}_{3}$ causes severe phase separation ${ }^{19-21}$. $\mathrm{TiTe}_{2}$ has the same HEX lattice as $\mathrm{Sb}_{2} \mathrm{Te}_{3}$, but a $\sim 11 \%$ lattice parameter (a) mismatch with $\mathrm{Sb}_{2} \mathrm{Te}_{3}$ (ref. 22). Still it is capable of forming superlattice with $\mathrm{Sb}_{2} \mathrm{Te}_{3}$ (ref. 23). To understand this unique and unexpected behaviour of TST, we performed firstprinciples calculations (see Methods for details).

The ST belongs to the $R \overline{3} m$ space group and is composed of HEX close-packed quintuple atomic layers along the c-axis (Fig. 4a) ${ }^{24}$. Rather than modelling the exact experimental composition, we calculate the formation energy of a single $\mathrm{Ti}$
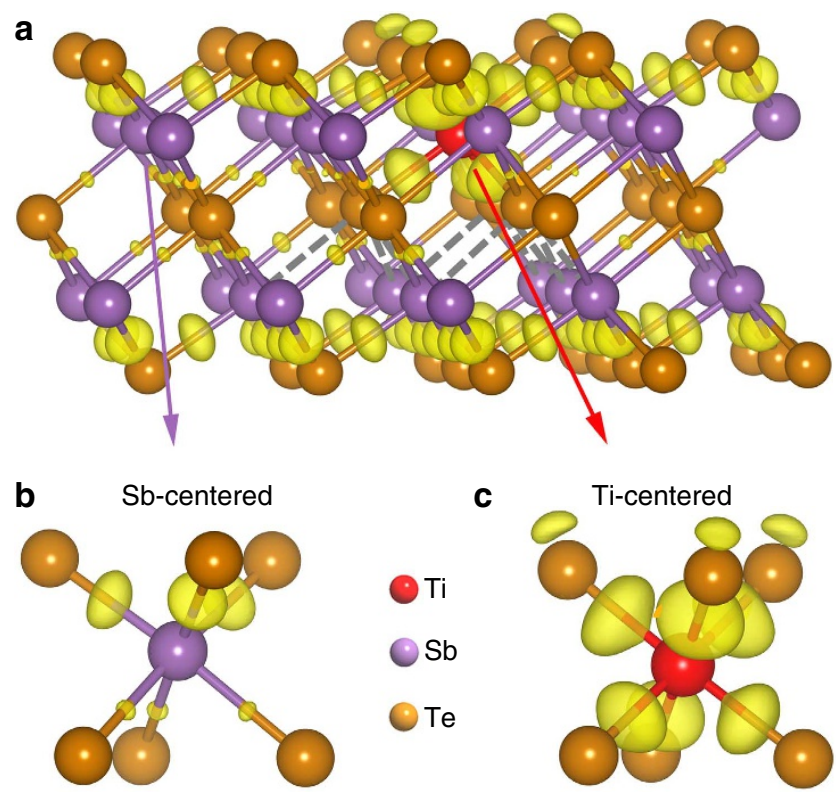

Figure 4 | Bonding chemistry of crystalline $\mathbf{T i}_{\mathbf{0 . 4}} \mathbf{S b}_{\mathbf{2}} \mathbf{T e}_{\mathbf{3}}$. (a) Hexagonal lattice of $\mathrm{Ti}_{0.4} \mathrm{Sb}_{2} \mathrm{Te}_{3}$ with charge density difference. The differences are calculated with respect to that of a superposition of isolated atoms. The isosurface (transparent yellow area) shows electron pileup mostly at the bonds. The isosurface value is fixed at +0.005 e $a_{0}^{-3}$ ( $a_{0}=$ bohr). (b) The Sb-centred atomic motif has three strong bonds and three weak bonds. (c) The Ti-centred atomic motif has six strong bonds and no weak bond. The formation of six strong Ti-Te bonds weakens some of the adjacent Te-Sb bonds (dashed line in (a)). 
atom at various ST lattice sites in a 135 -atom supercell to examine the effects of introducing Ti. The most stable site for the $\mathrm{Ti}$ is to replace $\mathrm{Sb}\left(\mathrm{Ti}_{\mathrm{Sb}}\right)$ with six Te nearest neighbours (Supplementary Fig. 5). X-ray photoelectron spectroscopy (XPS) analyses (Supplementary Fig. 5) confirm this Ti-Te bonding. To identify the bonding chemistry between $\mathrm{Ti}$ and Te, Fig. 4 shows the charge density difference $(\mathrm{CDD})^{25}$ : in the Sb-centred atomic motif (Fig. $4 \mathrm{~b}$ ), there are three strong and three weak bonds ${ }^{26}$. In contrast, in the TCAM (Fig. 4c), all the six bonds between Ti and $\mathrm{Te}$ are strong (covalent) bonds with noticeable charge accumulation at the bond centre similar to $\mathrm{TiTe}_{2}$ (ref. 27). Ti has the $d^{2} s^{2}$ electronic configuration; the strong covalent interaction is originated from the overlapping between $\mathrm{Ti}-3 d$ and Te-5p orbitals ${ }^{27}$. This covalent bonding may account for the rigidity of the TCAMs during phase transition. To compensate for the local rigidity, $\mathrm{Te}-\mathrm{Sb}$ bonds in the adjacent layer without the $\mathrm{Ti}$ are significantly elongated and weakened, as indicated in Fig. 4a by dashed lines. These weakened bonds may be deformed to accommodate the $11 \%$ lattice mismatch with ST. They may also be ruptured to break the long-range order during amorphization, leading to a low-energy Reset operation as shown in Fig. 2.

Figure 5a shows the calculated atomic structure of a-TST by using the melt-quench molecular dynamics technique. Figure $5 b, c$ compare the coordination number $(\mathrm{CN})$ and bond angle distribution (BAD) between a-TST and a-GST. Note that CNs for Ge in a-GST are 3, 4 and 5, whereas those for Ti in a-TST are mainly 6 and 7, respectively. These can be contrasted to the ideal $\mathrm{CN}$ for rocksalt $=6$. We also notice that, with respect to the ideal $90^{\circ}$ rocksalt bond angle, the peak position of the bond angles for Ti decreases by $5^{\circ}$ to $85^{\circ}$, while that for Ge increases by $5^{\circ}$ to $95^{\circ}$. It has been established that a-GST has both tetrahedral Ge of $s p^{3}$ bonding $\left(109.5^{\circ}\right)$ and octahedral Ge of $p^{3}$ bonding $\left(90^{\circ}\right)^{16,28,29}$.
The $95^{\circ}$ Ge peak in Fig. $5 c$ is consistent with the coexistence of $s p^{3}$ and $p^{3}$ bonding. In comparison, the BAD peaks for $\mathrm{Sb}$ and $\mathrm{Te}$ in both a-GST and a-TST remain to be around $90^{\circ}$ (see inset in Fig. 5c). In contrast, the higher $\mathrm{CN}$ and less-than- $90^{\circ} \mathrm{BAD}$ of $\mathrm{Ti}$ suggest that the TCAMs in a-TST remain to be octahedrons with perhaps some distortions. Specifically, $42 \%$ of the $\mathrm{Ti}$ are in the form of $\mathrm{Ti}^{-} \mathrm{Te}_{6}$ or $\mathrm{Sb}-\mathrm{Ti}-\mathrm{Te}_{5}$ and another $41 \%$ are sevenfold coordinated. We speculate that the sevenfold motifs may be easier to transform to sixfold TCAMs on phase transition than the Ge motifs with $\mathrm{CN} \leq 5$. Thus, fewer bond breaking may be required, accompanied by considerably less structural changes, for the TCAMs in Fig. 5d to form nucleation centres for the subsequent growth of the c-phase. In this regard, we note that the structure ordering of growth-dominated AgInSbTe may take place by aligning Sb-centred octahetrons near the amorphous to crystalline boundary ${ }^{30}$. The Sb-centred octahetrons in a-TST, around TCAMs as the nucleation centres, may also be easily aligned to realize long-range order.

One may also compare the crystallization speed in terms of the $A B A B$-type four-membered rings, where $A=(\mathrm{Ge}$ and $\mathrm{Sb})$ or ( $\mathrm{Ti}$ and $\mathrm{Sb}$ ) and $\mathrm{B}=\mathrm{Te}$. These distorted four-membered rings, proposed by Akola et al. ${ }^{12}$ and Hegedüs et al. ${ }^{14}$ to resemble the chemically ordered structural blocks of the rocksalt phase, may serve as the nucleation sites, whose rapid growth and accompanied reorientation aided by a large concentration of cavities may explain the observed rapid crystallization of the ordered phase. Figure $6 a$ shows that, among all the fourmembered rings, a-TST has a larger number of ABAB rings than those of a-GST. Furthermore, inset in Fig. 6a shows that, per Ti (Ge) atom, the number of $\begin{gathered}T_{i}-T_{j} \\ T e\end{gathered} T_{i(S b)}$ rings is twice more than that of $G_{e}-T_{1} e$
$T e-G e(S b)$ rings. This means $\mathrm{Ti}$ is obviously more likely to form four-membered rings than $\mathrm{Ge}$ does. All these analyses

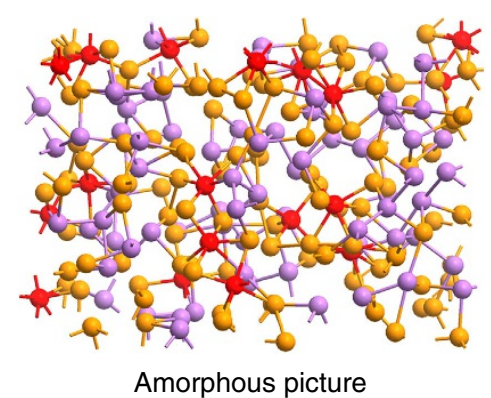

C

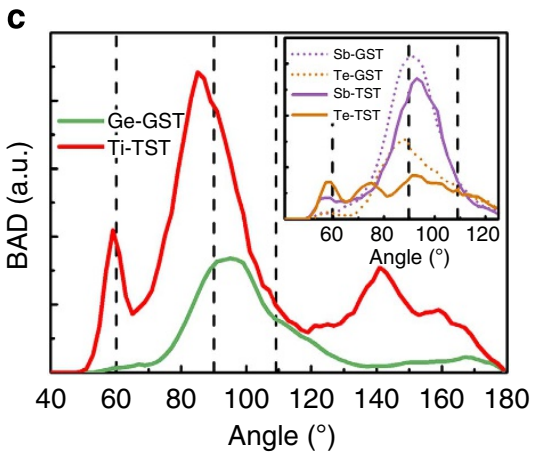

b

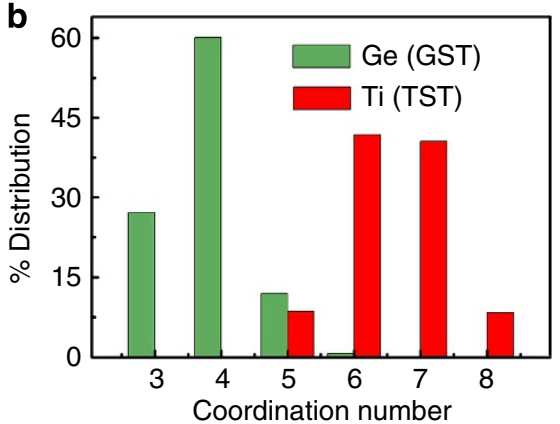

d

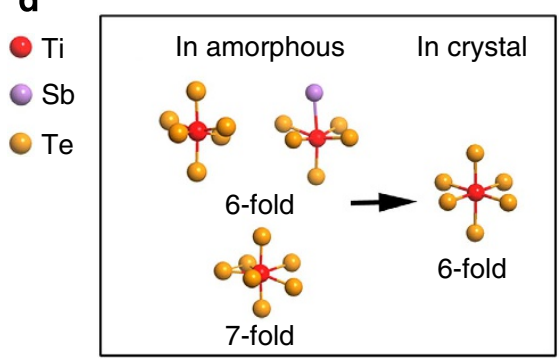

TCAM comparison

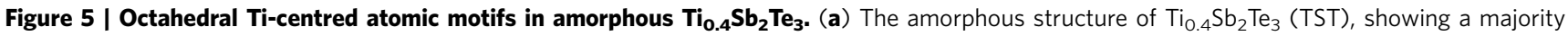
of the Ti, are bonded with Te and sixfold coordinated in an octahedral-like geometry. (b) The coordination number distribution of Ge and Ti in the respective amorphous model. (c) The bond angle distributions (BADs) around $\mathrm{Ge}$ in amorphous $\mathrm{Ge}_{2} \mathrm{Sb}_{2} \mathrm{Te}_{5}$ (GST) and Ti in amorphous TST. Inset shows the BADs around Sb and Te in amorphous GST and TST, respectively. Vertical dash lines indicate the positions of 60, 90 and $109.5^{\circ}$. (d) Proposed Ti-centred atomic motifs (TCAMs) in amorphous and crystalline TST. 

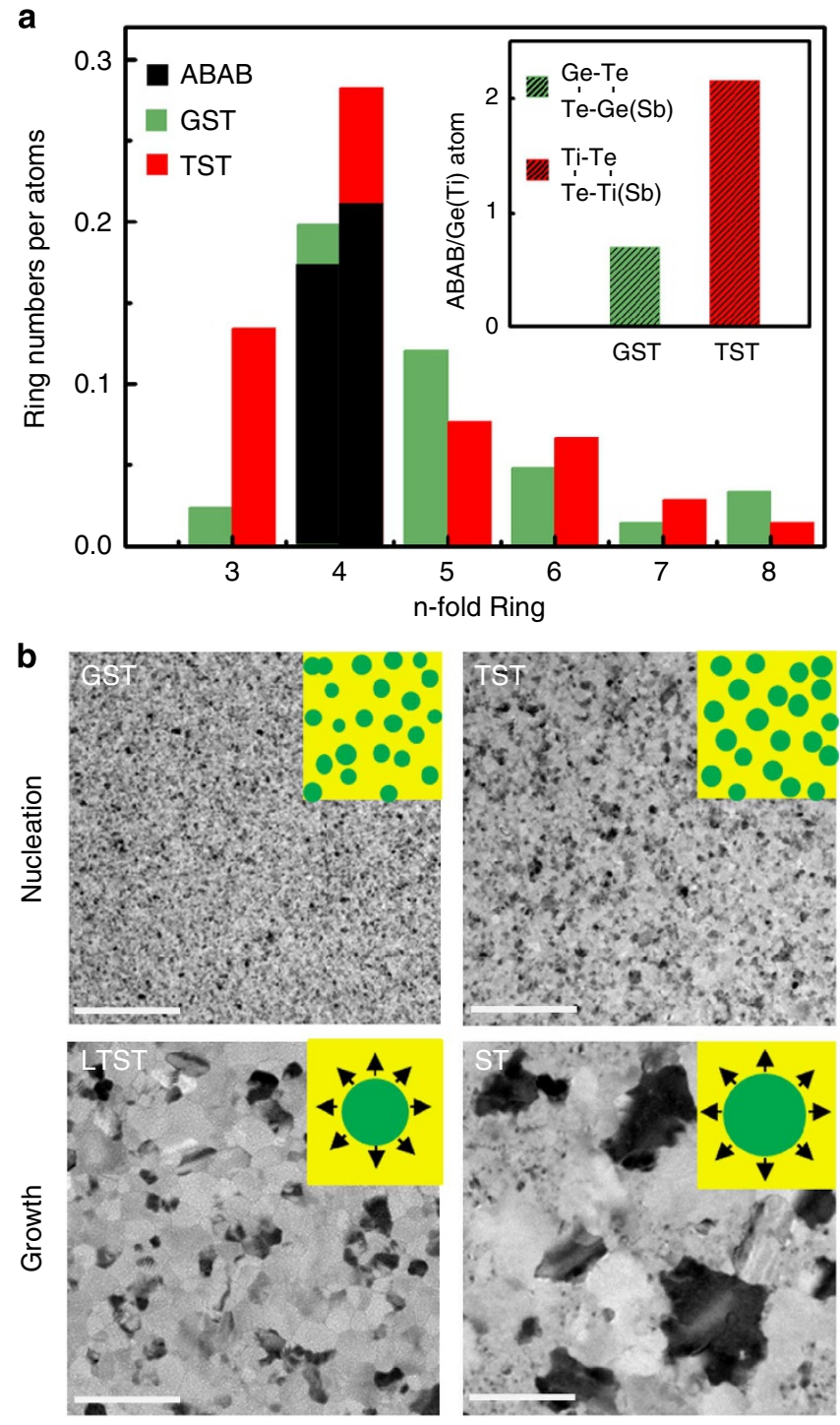

Figure 6 | Nucleation-dominated crystallization mechanism of

$\mathbf{T i}_{\mathbf{0 . 4}} \mathbf{S b}_{\mathbf{2}} \mathbf{T e}_{\mathbf{3}}$. (a) The $\mathrm{n}$-fold ring distributions of amorphous $\mathrm{Ge}_{2} \mathrm{Sb}_{2} \mathrm{Te}_{5}$ (GST) (green) and amorphous $\mathrm{Ti}_{0.4} \mathrm{Sb}_{2} \mathrm{Te}_{3}$ (TST) (red). The four-membered $A B A B$ rings are highlighted: $A=(G e$ and $S b)$ or $(T i$ and $S b)$ and $B=T e$. Inset shows the respective $A B A B$ ring (containing 1 or $2 \mathrm{Ge}(\mathrm{Ti})$ ) number per $\mathrm{Ge}(\mathrm{Ti})$ atom. (b) The TEM images of GST, TST, low-concentration $\mathrm{Ti}$ doped $\mathrm{Sb}_{2} \mathrm{Te}_{3}$ (LTST) and $\mathrm{Sb}_{2} \mathrm{Te}_{3}$ (ST) annealed at $250^{\circ} \mathrm{C}$ for $2 \mathrm{~min}$. All the scale bars correspond to $200 \mathrm{~nm}$.

consistently suggest that $\mathrm{Ti}$ doping increases the probability and the number of the nucleation centres. Hence, crystallization of the TST should be a nucleation-dominated process, which is also the case for GST (Fig. 6b (first row)).

Time-dependent in situ electron beam irradiation experiment confirms this nucleation-dominated mechanism for the crystallization of a-TST (Supplementary Fig. 6). Figure 6b (first row) shows that, in both GST and TST, the grain sizes are on the order of $10-15 \mathrm{~nm}$. In contrast, Fig. 6b (second row) shows that low-concentration Ti-doped ST (LTST) and pure ST exhibit a growth-dominated mechanism with much larger grain size of $50-160 \mathrm{~nm}$. Diminishing grain size is known to increase the interface-area-to-volume ratios to promote heterocrystallization at the grain boundaries, further speed up the crystallization $^{8}$.
In summary, a combined experimental and theoretical study reveals a new approach to drastically increase the speed, while decreasing the power, of PCM. These improvements are achieved, without having to rely on any complicated device fabrication and/ or programming method, as often being done now. Rather, it is realized by introducing robust TCAMs. We believe the TCAMs may play the role to pin the local structures in both a- and c-ST phases. They act as nucleation centres during the phase transition to avoid substantial atomic rearrangement, which in turn facilitates faster and lower-energy phase transitions. We expect the speed/power to be further increased/decreased significantly on a thorough investigation to optimize the composition of the Ti-Sb-Te. In this regard, a comprehensive model, which is not limited to the possible effect of nucleation centres but can also account for the interactive rearrangement of all the atoms, will be a great help. As the PCM dimension further scales, it is likely that the Ti-Sb-Te alloys will be more advantageous than the Ge-Sb-Te alloys to realize DRAM-like device applications.

\section{Methods}

PCM cell fabrication and electrical measurement. T-shaped PCM cell with $D=190(80) \mathrm{nm}$ tungsten plug BEC was fabricated using $0.13 \mu \mathrm{m}$ CMOS technology. The $\sim 170 \mathrm{~nm}$-thick TST film was deposited by co-sputtering of pure Ti and ST targets, and GST film with the same thickness was deposited by sputtering pure GST target. The $\sim 15 \mathrm{~nm}$-thick TiN and $\sim 300 \mathrm{~nm}$-thick Al films were used as top electrode in both GST and TST cells. All the electrical measurements were performed by using the Keithley $2400 \mathrm{C}$ source meter (measuring cell resistance), the Tektronix AWG5002B pulse generator (generating voltage pulse with a minimum width of $\sim 6 \mathrm{~ns}$ ), the homemade constant current driver (generating current pulse with a maximum magnitude of $\sim 10 \mathrm{~mA}$ ) and the Tektronix 7054 digital phosphor oscilloscope (measuring transient voltage drop across the cell when current pulse is applied).

Film characterization. The compositions of TST and GST films were measured by X-ray fluorescence spectroscopy using a Rigaku RIX 2100 system. For real-time observation of structure transition in pure ST and TST, in situ XRD was performed on 200-nm-thick films (deposited on Si substrate at room temperature) using PANalytical X'Pert PRO diffractometer with a $\mathrm{Cu} \mathrm{K} \alpha(\lambda=0.15418 \mathrm{~nm})$ radiation source. TST powders collected from the fragments of thick $(1 \sim 2 \mu \mathrm{m})$ TST amorphous film were used for DSC test to determine $T_{\mathrm{c}}$ and $T_{\mathrm{m}}$. The $20 \mathrm{~nm}$-thick ST and TST films were deposited on a series of Transmission Electron Microscope (TEM) sample supporting grids coated with carbon film. They were annealed at $250^{\circ} \mathrm{C}$ for $2 \mathrm{~min}$ to get the c-phase. These samples were studied using a FEI Tecnai F20 TEM in the bright field high-resolution TEM mode, selected area electron diffraction mode and scanning TEM mode. Nova 200 Nanolab Focused ion beam was used to fabricate the cross-sectional sample of the PCM cell for the TEM observation.

$\boldsymbol{A} \boldsymbol{b}$-initio theoretical simulation. Theoretical investigations employed the density functional theory (DFT) $)^{31}$. The Vienna ab-initio Simulations Package (VASP) ${ }^{32}$ was used. The projector augmented wave (PAW) pseudopotentials ${ }^{33}$ were used for electron-ion interactions. For the exchange-correlation energies between electrons, the Perdew-Burke-Ernzerhof (PBE) functional ${ }^{34}$ was used. A 207-atom cubic cell of GST and a 209-atom cubic cell of TST were simulated with periodic boundary conditions by NVT molecular dynamics (MD): to prepare the amorphous phase, first, the system was maintained at $3,000 \mathrm{~K}$ for $9 \mathrm{ps}$. Next, the temperature was lowered to $1,200 \mathrm{~K}$ for another $30 \mathrm{ps}$. The liquid was then quenched to $300 \mathrm{~K}$ at a quench rate of $-15 \mathrm{~K} \mathrm{ps}^{-1}$ to get the amorphous structure, which is maintained at $300 \mathrm{~K}$ for another $15 \mathrm{ps}$. The total simulation time is $114 \mathrm{ps}$. The energy cutoff is $178 \mathrm{eV}$ and the time step is $3 \mathrm{fs}$ for our MD.

For calculation of formation energy of a Ti atom in c-ST at its possible sites, a $13.01 \times 13.01 \times 31.26\left(\AA^{3}\right) 135$-atom supercell was used. A $2 \times 2 \times 1$ k-point mesh was used, but for MD simulation, only the $\Gamma$ point was used. The formation energy of $\mathrm{Ti}$ is given by ${ }^{35}$ :

$$
E^{\mathrm{f}}=E_{\mathrm{tot}}(\mathrm{TST})-E_{\mathrm{tot}}(\mathrm{ST})+\mu_{\mathrm{X}}-\mu_{\mathrm{Ti}},
$$

where $E_{\text {tot }}$ is the total energy per supercell with or without Ti. $\mu_{\mathrm{Ti}}$ is the chemical potential of $\mathrm{Ti}$ and $\mu_{\mathrm{X}}$ is the chemical potential of (Sb, Te) or 0 for $\mathrm{X}=\mathrm{Ti}_{\mathrm{Sb}}$ (Ti replacing $\mathrm{Sb}$ ), $\mathrm{Ti}_{\mathrm{Te}}$ ( $\mathrm{Ti}$ replacing $\mathrm{Te}$ ) or $\mathrm{Ti}_{\mathrm{i}}$ ( $\mathrm{Ti}$ intersitial). To maintain a stable ST requires:

$$
2 \mu_{\mathrm{Sb}}+3 \mu_{\mathrm{Te}}=E(\mathrm{ST})
$$

where $E(\mathrm{ST})$ is the total energy per formula unit of ST. 


\section{References}

1. Wuttig, M. Phase-change materials: towards a universal memory? Nature 4, 265-266 (2005).

2. Atwood, G. Phase-change materials for electronic memories. Science 321, 210-211 (2008).

3. Kim, I. S. et al. High performance PRAM cell scalable to sub-20 $\mathrm{nm}$ technology with below $4 \mathrm{~F}^{2}$ cell size, extendable to DRAM applications. Symp. on VLSI Tech. Dig. 203-204 (Honolulu, 2010).

4. Cheong, B. K. et al. Fast and scalable memory characteristics of Ge-doped SbTe phase change materials. Phys. Status Solidi B 10, 1985-1991 (2012).

5. Loke, D. et al. Breaking the speed limits of phase-change memory. Science 336, 1566-1569 (2012)

6. Kohara, S. et al. Structural basis for the fast phase change of: ring statistics analogy between the crystal and amorphous states. Appl. Phys. Lett. 89, 201910 (2006).

7. Wang, W. J. et al. Engineering grains of $\mathrm{Ge}_{2} \mathrm{Sb}_{2} \mathrm{Te}_{5}$ for realizing fast-speed, lowpower, and low-drift phase-change memories with further multilevel capabilities. IEDM Tech. Dig. 31.3.1-31.3.4 (San Francisco, 2012).

8. Wang, W. et al. Enabling universal memory by overcoming the contradictory speed and stability nature of phase-change materials. Sci. Rep. 2, 360 (2012).

9. Kang, M. J. et al. PRAM cell technology and characterization in $20 \mathrm{~nm}$ node size. IEDM Tech. Dig. 3.1.1-3.1.4 (Washington, 2011).

10. Sasago, Y. et al. Phase-change memory driven by poly-Si MoS transistor with low cost and high-programming gigabyte-per-second throughput. Symp. on VLSI Tech. Dig. 96-97 (Honolulu, 2011).

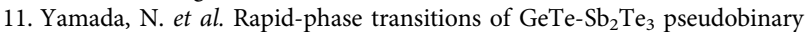
amorphous thin films for an optical disk memory. J. Appl. Phys. 65, 2849-2856 (1991).

12. Akola, J. \& Jones, R. O. Structural phase transitions on the nanoscale: The crucial pattern in the phase-change materials $\mathrm{Ge}_{2} \mathrm{Sb}_{2} \mathrm{Te}_{5}$ and GeTe. Phys. Rev. $B$ 76, 235201 (2007).

13. Kalikka, J. et al. Nucleus-driven crystallization of amorphous $\mathrm{Ge}_{2} \mathrm{Sb}_{2} \mathrm{Te}_{5}: \mathrm{A}$ density functional study. Phys. Rev. B 86, 144113 (2012).

14. Hegedüs, J. \& Elliott, S. R. Microscopic origin of the fast crystallization ability of Ge-Sb-Te phase-change memory materials. Nat. Mater. 7, 399-405 (2008).

15. Kolobov, A. et al. Understanding the phase-change mechanism of rewritable optical media. Nat. Mater. 3, 703-708 (2004).

16. Krbal, M. et al. Intrinsic complexity of the melt-quenched amorphous $\mathrm{Ge}_{2} \mathrm{Sb}_{2} \mathrm{Te}_{5}$ memory alloy. Phys. Rev. B 83, 054203 (2011).

17. Yin, Y., Sone, H. \& Hosaka, S. Characterization of nitrogen-doped $\mathrm{Sb}_{2} \mathrm{Te}_{3}$ films and their application to phase-change memory. J. Appl. Phys. 102, 064503 (2007).

18. Silva, J. L. F. Da, Walsh, A. \& Lee, H. Insights into the structure of the stable and metastable $(\mathrm{GeTe})_{\mathrm{m}}\left(\mathrm{Sb}_{2} \mathrm{Te}_{3}\right)_{\mathrm{n}}$ compounds. Phys. Rev. B 78, 224111 (2008).

19. Rao, F. et al. $\mathrm{Si}-\mathrm{Sb}-\mathrm{Te}$ materials for phase change memory applications. Nanotechnology 22, 145702 (2011)

20. Peng, C. et al. $\mathrm{Al}_{1.3} \mathrm{Sb}_{3} \mathrm{Te}$ material for phase change memory application. Appl. Phys. Lett. 99, 043105 (2011).

21. Raoux, S. et al. Effect of $\mathrm{Al}$ and $\mathrm{Cu}$ doping on the crystallization properties of the phase change materials SbTe and GeSb. J. Appl. Phys. 101, 044909 (2007).

22. Greenaway, D. L. \& Nitsche, R. Preparation and optical properties of group IV $-\mathrm{VI}_{2}$ chalcogenides having the $\mathrm{CdI}_{2}$ Structure. J. Phys. Chem. Solids 26, 1445-1458 (1965)

23. Schmid, B. et al. Synthesis of $\left(\mathrm{Sb}_{2} \mathrm{Te}_{3}\right)_{\mathrm{X}}\left(\mathrm{TiTe}_{2}\right)_{\mathrm{Y}}$ superlattices. ICT Thermo 270-272 (2005)

24. Anderson, T. L. \& Krause, H. B. Refinement of the $\mathrm{Sb}_{2} \mathrm{Te}_{3}$ and $\mathrm{Sb}_{2} \mathrm{Te}_{2} \mathrm{Se}$ structures and their relationship to nonstoichiometric $\mathrm{Sb}_{2} \mathrm{Te}_{3-\mathrm{y}} \mathrm{Se}_{\mathrm{y}}$ compounds. Acta Cryst B30, 1307-1310 (1974).

25. Kolobov, A. et al. Vacancy-mediated three-center four-electron bonds in GeTe$\mathrm{Sb}_{2} \mathrm{Te}_{3}$ phase-change memory alloys. Phys. Rev. B 87, 165206 (2013).

26. Kolobov, A. et al. Why phase-change media are fast and stable: a new approach to an old problem. Jpn J. Appl. Phys. 44, 3345-3349 (2005).
27. Kim, Y. et al. Electronic structures and chemical bonding of $\mathrm{TiX}_{2}(\mathrm{X}=\mathrm{S}, \mathrm{Se}$, and Te). Jpn J. Appl. Phys. 37, 4878-4883 (1998).

28. Caravati, S. et al. Coexistence of tetrahedral- and octahedral-like sites in amorphous phase change materials. Appl. Phys. Lett. 91, 171906 (2007).

29. Shportko, K. et al. Resonant bonding in crystalline phase-change materials. Nat. Mater. 7, 653-658 (2008)

30. Matsunaga, T. et al. From local structure to nanosecond recrystallization dynamics in AgInSbTe phase-change materials. Nat. Mater. 10, 129-134 (2011).

31. Hohenberg, P. \& Kohn, W. Inhomogeneous electron gas. Phys. Rev. 136, B864-B871 (1964).

32. Kresse, G. \& Hafner, J. Ab initio molecular dynamics for liquid metals. Phys. Rev. B 47, 558-561 (1993).

33. Blöchl, P. E. Projector augmented-wave method. Phys. Rev. B 50, 17953-17979 (1994).

34. Perdew, J. P., Burke, K. \& Ernzerhof, M. Generalized gradient approximation made simple. Phys. Rev. Lett. 77, 3865-3868 (1996).

35. Zhang, S. B. \& Northrup, J. E. Chemical potential dependence of defect formation energies in GaAs: Application to Ga self-diffusion. Phys. Rev. Lett. 67, 2339-2342 (1991).

\section{Acknowledgements}

This work was supported by Strategic Priority Research Program of Chinese Academy of Sciences (XDA09020402), National Key Basic Research Program of China (2013CBA01902, 2011CBA00607, 2011CB932804, 2010CB934300), National Integrate Circuit Research Program of China (2009ZX02023-003), National Natural Science Foundation of China (61076121, 61176122, 61106001, 61261160500, 61376006, 11104109, 11374119), Science and Technology Council of Shanghai (11DZ2261000, 12nm0503701, 12QA1403900, 13ZR1447200, 13DZ2295700). Professor S.Z. was supported by the US Department of Energy under Grant No. DE-SC0002623. The supercomputer time was provided by HPCC at JLU.

\section{Author contribution}

M.Z. and S.L. prepared the film samples and carried out the in situ XRD, DSC, XPS, in situ electron irradiation and TEM experiments and so on. M.Z. and F.R. fabricated the PCM cell samples and carried out electrical measurements. M.X. and X.L. performed the AIMD calculations. F.R., X.L. and S.Z. carried out theoretical analysis and together wrote this paper with help from all co-authors. The project was initiated and conceptualized by F.R. and Z.S.

\section{Additional information}

Supplementary Information accompanies this paper at http://www.nature.com/ naturecommunications

Competing financial interests: The authors declare no competing financial interest.

Reprints and permission information is available online at http://npg.nature.com/ reprintsandpermissions/

How to cite this article: Zhu, M. et al. One order of magnitude faster phase change at reduced power in Ti-Sb-Te. Nat. Commun. 5:4086 doi: 10.1038/ncomms5086 (2014)

This work is licensed under a Creative Commons AttributionNonCommercial-NoDerivs 4.0 Unported License. The images or other hird party material in this article are included in the article's Creative Commons license, unless indicated otherwise in the credit line; if the material is not included under the Creative Commons license, users will need to obtain permission from the license holder to reproduce the material. To view a copy of this license, visit http://creativecommons.org/licenses/by-nc-nd/4.0/ 